\title{
Les mots en crue : poésie et écologie dans le bassin versant du fleuve Sénégal
}

Swelling words: poetry and ecology in the River Senegal's watershed

\section{Marie Lorin}

\section{(2) OpenEdition}

\section{Journals}

Édition électronique

URL : https://journals.openedition.org/clo/8438

DOI : $10.4000 /$ clo.8438

ISSN : 2266-1816

Éditeur

INALCO

\section{Édition imprimée}

Date de publication : 30 juin 2020

Pagination : 101-122

ISBN : 978-2-85831-392-1

ISSN : 0396-891X

Référence électronique

Marie Lorin, «Les mots en crue : poésie et écologie dans le bassin versant du fleuve Sénégal », Cahiers de littérature orale [En ligne], 87 | 2020, mis en ligne le 16 septembre 2021, consulté le 09 novembre 2022. URL : http://journals.openedition.org/clo/8438 ; DOI : https://doi.org/10.4000/clo.8438

\section{(c) (i) \&}

Creative Commons - Attribution - Pas d'Utilisation Commerciale 4.0 International - CC BY-NC 4.0 https://creativecommons.org/licenses/by-nc/4.0/ 


\title{
Les mots en crue : poésie et écologie dans le bassin versant du fleuve Sénégal
}

\author{
Marie LORIN \\ Inalco, Llacan \\ "A poem is stored energy, a formal turbulence, a living thing, a swirl in \\ the flow. \\ Poems are part of the energy pathways which sustain life. \\ Poems are a verbal equivalent of fossil fuel (stored energy), but they \\ are a renewable source of energy, coming, as they do, from those ever \\ generative twin matrices, language and imagination." \\ William Rueckert, Literature and Ecology
}

\section{Introduction}

En 1978, William Rueckert, universitaire américain et théoricien de la littérature, s'empare de l'écologie pour renouveler son approche critique et sa position d'enseignant en littérature en posant pour la première fois le concept d'écocritique. Selon lui, le poème porterait une énergie renouvelable et infinie passant d'un être à un autre, une énergie relationnelle apte à faire dialoguer la diversité des vivants, une écologie, une « science des relations des organismes avec le monde environnant, c'est-à-dire, dans un sens large, la science des conditions d'existence ${ }^{1} \gg$. Cet article interrogera cette hypothèse d'une poésie comme écologie à partir d'un exemple précis : le pékâne, la poésie orale en peul des pêcheurs de la Vallée du Fleuve Sénégal, région située à cheval entre Sénégal et Mauritanie.

1. Première définition de l'écologie donnée par Haeckel en 1866 (Haeckel, 1866). 
Le fleuve Sénégal prend sa source en Guinée, remonte au Mali où il est alimenté par de nombreux affluents qui le font gagner en force, devient un espace frontière entre le Sénégal et la Mauritanie, puis termine sa course en se jetant dans l'océan Atlantique. Se déployant en grande partie en région sahélienne, le fleuve entre en crue à l'issue d'une saison des pluies de trois à quatre mois (de juin/juillet à septembre) qui succède à une longue saison sèche (d'octobre à juin) lors de laquelle l'aridité et la chaleur semblent avoir progressivement jeté toute forme de vie dans une sorte de léthargie. La pluie d'abord, la crue ensuite, viennent alors redonner vie. L'eau inonde la vallée du fleuve remplissant rivières, mares, marigots, reverdissant herbes et arbres, animant humains et animaux. La crue est aussi un moment de grand danger, les inondations pouvant ravager les constructions et emporter hommes et biens. Elle peut donc être synonyme de vie comme de mort, susciter espoir autant qu'inquiétude. L'eau est une énergie conflictuelle : le retour de la vie sur une terre desséchée, la puissance dévastatrice des flots. Dans la région du Foûta Tôro ${ }^{2}$, une catégorie sociale particulière est chargée de la gestion du fleuve et ses membres sont considérés par le reste de la population ${ }^{3}$ comme les « maîtres des eaux ». Ils pêchent les poissons à bord de pirogues et à l'aide de filets, mais sont aussi chargés de gérer le fleuve : protéger les riverains non pêcheurs (contre les crocodiles, dans la prévention et le secours de noyade), et aussi protéger le fleuve des riverains (interdiction de polluer l'eau, gestion raisonnée des ressources de poissons). Cette communauté de pêcheurs accorde une place extrêmement importante à la poésie, qu'elle soit traditionnelle ou contemporaine, déclamée ou chantée.

Il s'agira de comprendre comment le pékâne, poésie traditionnelle et encore très dynamique des pêcheurs, contribue à affirmer une vision émique de

2. Les enquêtes dont cet article est issu se sont déroulées de 2010 à 2020 (une quinzaine de séjours sur le terrain) dans le département de Podor, au Nord Sénégal. Podor, est aussi le nom de la ville chef-lieu du département du même nom. Cette ville est située sur l'île à Morfil, bande de terre large de $150 \mathrm{~km}$ et d'environ $30 \mathrm{~km}$ de large entre le fleuve Sénégal et l'un de ses plus gros affluents, le Doué. Lors de la crue, l'île à Morfil devient un archipel, de nombreux villages s'y situant étant entourés d'eau, la circulation se fait alors essentiellement en pirogue.

3. Cette région est organisée en catégories sociales. Les pêcheurs, maîtres des eaux (en poulâr, subalbe au pluriel, cuballo au singulier) font partie des hommes libres comme les éleveurs et les religieux. Les artisans constituent la deuxième grande strate sociale : griots, boisseliers, tisserands, etc. La troisième catégorie sociale est constituée par les descendants de captifs, aujourd'hui majoritairement affranchis et pouvant occuper des postes à responsabilité mais subissant souvent une forme de marginalisation sociale. 
l'écosystème fluvial en faisant entendre la façon dont sont tissées des relations entre les mondes humains, végétaux, animaux, magiques. Le fleuve n'y est pas considéré comme une ressource à exploiter, mais comme une entité vivante dont le mouvement crée une énergie relationnelle, fait coexister une diversité de mondes qui dialoguent. Or ce dialogue est possible grâce à une capacité d'écoute du monde fluvial, spécifique aux pêcheurs et sur laquelle repose leur maîtrise des eaux du fleuve, la maîtrise de ses mouvements dont la poésie se fait l'écho.

À partir des positions de Steven Feld (Feld, 1996) sur l'acoustémologie, définie par lui comme «le potentiel du savoir acoustique, du son comme condition du savoir et pour savoir, de la présence et conscience soniques comme des forces puissantes qui façonnent la manière dont les gens donnent sens à leurs expériences », l'article analysera la façon dont l'écoute du fleuve qui engage de la part des pêcheurs une corporéité spécifique, celle de l'immersion dans l'eau et de la plongée, est au cœur de la composition poétique et des savoirs écologiques que cette poésie déploie.

\section{L'écoute comme savoir}

Cette poétique relationnelle est au cœur du pékâne, la forme de poésie la plus répandue chez les pêcheurs. Le mot pékâne vient de la racine verbale feekaade qui signifie « clamer, crier, appeler à haute voix » (Seydou, 1998). La voix est donc centrale dans la définition du genre. Il s'agit d'une poésie chantée a capella essentiellement par des hommes appartenant à la catégorie sociale des pêcheurs lors de performances qui se déroulent dans différents contextes festifs liés à la pratique de la pêche ${ }^{4}$. Plusieurs études (Sow, 2009 ; Sy, 1978 ; Ndiaye, 2005 ) ont décrit les cérémonies de chasse aux crocodiles (fiffire en poulâr) qui donnaient lieu à des performances de pékâne qui avaient alors pour fonction d'encourager les pêcheurs dans leurs combats. Aujourd'hui les crocodiles sont devenus rares; ce genre de cérémonie et donc ce type de performance aussi. Mais le pékâne a subsisté et se déploie toujours dans des festivals, des soirées culturelles, des démonstrations de régates, des évènements familiaux ou politiques. Les performances dont sont issus les extraits présentés dans cet article ont été enregistrées dans des contextes divers. Deux d'entre elles ont été enregistrées lors d'une démonstration organisée par mon collaborateur pour me faire découvrir le pékâne. Elles ont eu lieu au bord puis sur le fleuve Sénégal. Des pêcheurs présents

4. Il existe une femme qui chante le pékâne, mais cela semble un phénomène extraordinaire, la norme est que le pékâne soit un chant d'homme. 
sont intervenus. D'autres chants ont été enregistrés lors d'un festival culturel, le « Blues du fleuve », organisé à Podor et qui réunit plusieurs chanteurs de pékâne mais aussi de nombreux artistes de la région, du Sénégal et de la Mauritanie. Enfin d'autres performances ont été enregistrées lors de fêtes villageoises où les chanteurs encourageaient des pagayeurs engagés dans des démonstrations de régates. Dans ce dernier cadre, les villageois appartenant à la communauté des pêcheurs constituaient l'auditoire et les potentiels répondants. Une performance de pékâne est en effet une co-construction entre un chanteur ${ }^{5}$ et un auditoire composé en grande partie de pêcheurs dont certains interviennent au cours du chant. Ces interventions s'appellent sappaade ${ }^{6}$ et peuvent arriver à tout moment dans la performance. Le chanteur chante, les pêcheurs l'interrompent en déclamant des incantations et/ou en narrant leurs exploits (combats contre des crocodiles, des hippopotames). Ces performances ont des durées variables, de quelques minutes à plusieurs dizaines de minutes, et peuvent mettre en scène un ou plusieurs chanteurs qui se relaient.

De quoi est-il question ? La réponse (partielle) est donnée par deux vers qui reviennent régulièrement :

hannde dee ganndo maayo

aujourd'hui en connaisseur du fleuve

ma mi haal maayo

je vais parler du fleuve

Cet extrait définit a minima une position et un programme : le chanteur connaît le fleuve, et c'est parce qu' il connaît le fleuve qu'il peut chanter. Le pékâne est un chant qui parle du fleuve. Le fleuve est ce qui donne légitimité à chanter et ce qui doit être chanté. Or le fleuve n'est pas qu'un objet du chant, il est une entité avec laquelle on dialogue, ce qui suppose écoute et parole, et donc une double sensibilité. D’abord, la sensibilité du chanteur qui écoute le fleuve et appelle son auditoire à écouter le fleuve. Une courte phrase revient ainsi plusieurs fois dans chaque performance : «kedodaa maayo/écoute le fleuve ». Ousmane Niang, chanteur de pékâne et pêcheur explique ainsi l'écoute à laquelle ces vers invitent :

5. Les chanteurs de pékâne dédient leur vie à la poésie dont ils tirent des revenus, beaucoup d'entre eux pratiquent d'autres activités pour augmenter leurs revenus : pêche ou agriculture par exemple.

6.sappaade, racine verbale qui signifie « pointer l'index, mettre au défi », SEYdou, 1998. 
Si tu es fils de Tiouballo et que tu es couché la nuit, tu entends alors les bruits du fleuve et tu communiques avec lui car ton père et ta mère y ont puisé des choses pures et te les ont données. Si tu es couché tranquillement, tu entendras la quiétude du fleuve. Qu'est ce qui calme le fleuve ? On peut calmer le fleuve jusqu'à le rendre doux. On peut le réveiller jusqu'à ce qu'il s'agite. Si le fleuve est calme tu entends ce qu'il dit. Si le fleuve est agité, tu entends ce qu'il dit. Cela vient de l'enfance ${ }^{7}$.

L'écoute est au cœur des savoir-faire des pêcheurs. Tout est sonore dans le fleuve si on sait écouter : les courants, les bruits des poissons, oiseaux, des différentes créatures y habitant. Cette écoute permet d'anticiper les difficultés et les opportunités de la journée à venir : la présence ou non de poissons, les dangers éventuels qui pourront se présenter. La nuit est le moment privilégié de cette écoute. Alors qu'on ne voit rien, qu'on est dans son lit, c'est l'écoute qui est « la condition du savoir » (Feld, 1996). Le pékâne est l'écho de cette écoute spécifique qui est le préalable au dialogue.

\title{
La crue
}

Qu'écoute-t-on ? D'abord les mouvements de l'eau, le rythme du fleuve. Le pékâne appelle l'auditoire à rentrer dans une sensorialité particulière : celle de l'eau et de ses mouvements. La région du fleuve Sénégal voit des extrêmes climatiques se succéder : aridité lors de la saison sèche, inondations, rivières et mares remplies, reverdissement général lors de la saison des pluies. Le pékâne fait entendre ce basculement du vivant, il appelle régulièrement les auditeurs à écouter les mouvements de l'eau, à adopter son rythme, à devenir aquatique :

\author{
kedodaa maayo \\ tu écoutes le fleuve \\ nganndaa yoo nde laana weli e maayo \\ tu sais quand la pirogue est agréable sur le fleuve \\ demminaare oo ranwa majon majii funnaange wempeyere \\ wuppa yuufa jonya waraango
}

\footnotetext{
7. Extrait du film Kedodaa maayo/Écoute le fleuve réalisé par Franck Guillemain et Marie Lorin, 2020.
} 
la fin de la saison sèche blanchit le monde, de petits éclairs strient le ciel d'Est, la vague libère de l'écume et repousse les rapides

so ile naatii e kebbe

quand les crues pénètrent entre les cram-crams ${ }^{8}$

hakkunde rewo e worgo tay a ko pucci e coweeji ngonnoo

entre nord et sud, elle brise l'espace des chevaux et des bœufs

\section{ko ndeen labale e gini centee liggee hirkeeji kabbitee njowee}

c'est le moment où l'on retire mors et brides pour les suspendre, où l'on retire les selles pour les accrocher

\section{heddoo Siwolumgaal laana}

Ne reste que Siwoloumgâl la pirogue

\section{meetoo bonnaani}

à la forme parfaite ${ }^{9}$

Le rythme de l'extrait se fait l'écho du basculement qu'opère la crue : la montée des eaux consécutive à l'arrivée de la pluie, la fin provisoire de la vie terrestre, la nécessité d'adopter un mode de vie aquatique, d'adopter le rythme de l'eau. L'expression « kedodaa maayo/écoute le fleuve » est directement suivie d'un rappel des qualités de la pirogue : «nganndaa yoo nde laana weli e maayo/tu sais quand la pirogue est agréable sur le fleuve ». Écouter le fleuve, c'est apprécier les possibilités d'y voguer, reconnaître la beauté de la pirogue comme mode de transport certes, mais aussi comme possibilité de vie avec le fleuve, comme mode de relation possible. Le rythme joue sur des effets d'accélération liés à la montée des eaux : « demminaare oo ranwa majon majii funnaange wempeyere wuppa yuufa jonya waraango/la fin de la saison sèche blanchit le monde, de petits éclairs strient le ciel d'Est, la vague libère de l'écume et repousse les rapides ». Cette accélération est perceptible dans l'expression majon/maji qui crée un effet de dédoublement, d'échos. Ces éclairs débutent à l'est, ils annoncent le début du jour, le recouvrement progressif de tout le ciel par l'orage. Mais dans le chant,

8. Cenchrus biflorus : herbacées épineuses dont les graines piquent et s'accrochent aux vêtements.

9. Extrait issu d'une performance de Souleymâne Maal enregistrée en octobre 2011 à Doué, Sénégal. 
les effets de l'orage se répercutent directement sur le fleuve et les mouvements de l'eau, comme si le poète traduisait les mouvements du ciel par les réactions du fleuve. Ce ne sont pas les nuages qui se brisent pour faire tomber la pluie, mais directement les vagues qui libèrent l'écume et provoquent de forts courants. La pluie est implicite.

Rythmiquement, la phrase se fait l'écho des effets du ciel sur le fleuve. À l'arrivée de la saison des pluies (demminaare oo ranwa), le chanteur prend son temps : il allonge particulièrement la voyelle longue -aa- et ajoute une onomatopée qu'il allonge aussi -oo-. À ce début haut mais lent succède ensuite une phrase qui ne fait que gagner en rapidité. La phrase s'agite, les sons se bousculent, enchaînant allitérations (-w-, -p-), assonances (-e-, -a-, -u-, -o-) et voyelles courtes alors que les longues sont peu marquées. La succession des sons épouse le rythme des courants (waraango). Cette accélération est suivie d'un vers court mais dense annonçant l'arrivée de la crue : « so ile naatii e kebbe/quand les crues pénètrent entre les crams-crams ». La force de la crue est condensée dans la puissance d'un seul vers court mais qui crée un basculement : les vagues (wempeyere) et les courants (waraango) se rassemblent en un phénomène dont la puissance balaie tout. Un nouveau cycle s'ouvre alors, et de nouveau, la phrase s'accélère : « hakkunde rewo e worgo taya ko pucci e coweeji ngonnoo/entre nord et sud, elle brise l'espace des chevaux et des bœufs, ko ndeen labale e gini ceytee liggee hirkeeji kab6itee njowee/c'est le moment où l'on retire mors et brides pour les suspendre, où l'on retire les selles pour les accrocher ».

Seule l'évocation de la pirogue, louée là encore pour sa beauté (« meetoo bonnaani/à la forme parfaite »), permet de ramener une forme de calme rythmique : les voyelles sont particulièrement allongées, la précipitation n'est plus de mise, et c'est l'adéquation entre le fleuve et la pirogue qui est soulignée. La crue est donc le moment de la métamorphose, métamorphose de l'espace bien sûr, mais surtout métamorphose des êtres qui le vivent. La pirogue est un idéal, une façon de vivre l'eau et d'accompagner ses mouvements, de faire de la crue non pas un abandon de l'espace mais bien un moment de métamorphose.

\section{Le maelström}

Cette métamorphose produite par les mouvements de l'eau n'est pas uniquement présente lors de la crue ; un mouvement particulier de l'eau revient à plusieurs reprises dans chaque performance et semble symboliser cette modification sensorielle familière aux pêcheurs à laquelle appelle le pékâne. Ce mouvement, c'est le maelström, un tourbillon de l'eau (jiriile en poulâr) : 
kedodaa maayo

écoute le fleuve

\section{kedodaa jiriile maayo kedodaa jannjaali}

écoute les maelströms du fleuve écoute ses joies

Un maelström fait a priori appel à la vue, mais ici l'auditeur est appelé à l'écouter, comme il doit écouter le fleuve et ses joies, dont les tourbillons seraient une manifestation. Pour les pêcheurs que j'ai interrogés, le maelström revêt un second sens puisqu'il désigne aussi la capacité d'action des pêcheurs dans le fleuve et leurs connaissances personnelles, magiques pour la plupart. Or ces capacités s'expriment d'abord par le déploiement d'une sensorialité particulière, à la fois visuelle et auditive. Le maelström est le point de passage entre une sensibilité terrestre et celle, aquatique, mobilisée par les pêcheurs lorsqu'ils vont sous l'eau explorer les fonds du fleuve. Les pêcheurs sont des apnéistes : ils nagent, font de la pirogue, mais surtout ils plongent. Ils doivent le faire fréquemment pour s'occuper de leurs filets parfois retenus au fond de l'eau par des herbes ou des branchages. Cette pratique de l'apnée est une façon de mesurer leurs capacités, car le monde subfluvial est réputé dangereux et requiert des capacités spécifiques. Selon les riverains du fleuve et en particulier les pêcheurs, le fond du fleuve est habité par des créatures invisibles pour le non-initié mais agissantes, des génies du fleuve qui peuvent prendre des formes humaines ou animales mais qui sont à chaque fois plus-qu'humain ou plus-qu'animal. C'est ce monde que découvrent les pêcheurs en plongeant. Mais cette pratique de l'apnée modifie physiologiquement leurs capacités sensorielles. Certains reviennent avec l'ouie modifiée de ces voyages sous l'eau. Plusieurs histoires racontent les péripéties de personnes restées trop longtemps au fond du fleuve, parfois plusieurs jours et revenues sourdes mais clairvoyantes, capables d'agir sur le fleuve et ses créatures, d'obtenir ses bienfaits, de prévenir ses dangers. Établir un dialogue avec le fleuve, accéder à sa vie secrète passe donc dans la pratique par le dérèglement des sens si bien décrit par Rimbaud dans la Lettre du voyant : « Le Poète se fait voyant par un long, immense et raisonné dérèglement de tous les sens. » Les poètes pêcheurs se font eux aussi voyants et passeurs. Ils inventent une langue pour rendre audible cette sensorialité spécifique de l'eau, cette capacité métamorphique qui met en relations les mondes.

\section{Incantations}

En effet, si l'on écoute le fleuve, on lui parle aussi et la langue du pékâne relaie cette dimension grâce à la présence des incantations qui interviennent à plusieurs 
reprises dans les performances, soit par les chanteurs eux-mêmes, soit dans les interventions des pêcheurs. Les incantations sont très présentes dans la vie des pêcheurs. Elles servent à entrer en relation avec le fleuve, les animaux, les mondes invisibles, à se protéger, à pêcher, à chasser, à affronter les épreuves de la vie, à agir sur ces formes de vie. Langage performatif par excellence, les incantations peuvent paraître mystérieuses et sont difficilement traduisibles. Elles empruntent au poulâr, à l'arabe, au wolof et sont parfois dites dans des langues totalement inventées. Phrase ou ensemble de phrases courtes, elles s'apparentent en partie à des onomatopées qui imitent les mouvements des animaux ou le bruit des coups échangés, et elles déploient une musicalité très forte :

so alaa ko heddi so wonaa ngabu

si seul reste l'hippopotame

diwi hakkunde maayo

qui saute au milieu du fleuve

ada buli ene ardi e doole

tu as peur parce qu' il vient avec force

ada usta doole dee gila hakkunde mayo

tu diminues sa force depuis le milieu du fleuve

ada usta doole dee

tu diminues sa force

nducca nducca

ndouthia, ndouthia ${ }^{10}$

lakara ngabu

lakara ${ }^{11}$ l'hippopotame

[...]

maanaa

mânâ $\hat{a}^{12}$

doole ngasii ene heddii keynje

ses forces sont finies mais il lui reste ses pouvoirs magiques

10. Onomatopées.

11. Idem.

12. Idem. 
L'extrait ci-dessus relate un combat entre un pêcheur et un hippopotame. Le combat n'est pas physique : le pêcheur est sur la rive, l'hippopotame est au milieu du fleuve. L'affrontement est uniquement vocal, les incantations sont très courtes : nducca, nducca; lakara; maanaa. Leurs effets sont immédiats : « ada usta doole dee/tu diminues sa force ». L'hippopotame n'est pas une proie passive et possède lui aussi un langage magique, des capacités d'action qui ne passent pas nécessairement par la force physique pure (« doole ngasii ene heddii keynje/ses forces sont finies mais il lui reste ses pouvoirs magiques $\gg)$.

La particularité de l'énonciation des incantations dans le cadre d'une performance de pékâne réside dans leur aspect sonore et public. Le pékâne est l'un des seuls moments où les incantations sont dites devant autant de public, en dehors de leur champ d'action habituel. Elles peuvent être dites à haute voix dans le cadre de la transmission, lorsqu'un pêcheur apprend aux jeunes de sa famille la pratique de la pêche ou dans certaines cérémonies spécifiques ${ }^{13}$. Les incantations sont en partie héritées, et en partie créées. Selon Ibrahima Maal, Jaaltaabe de Podor, certaines incantations sont enseignées d'office à tous les jeunes de la famille afin qu'ils puissent s'assurer un minimum de protection notamment vis-à-vis du fleuve. Mais les incantations spécifiques à la pêche et nécessaires à l'approfondissement de cette pratique, sont enseignées seulement si le jeune en fait la demande. Les incantations sont alors mémorisées progressivement. Chaque pêcheur pratiquant le fleuve crée également de nouvelles incantations en fonction de ce qu'il a appris du fleuve, des situations et forces auxquelles il a dû faire face. Les incantations sont une façon de modéliser un savoir, le résultat d'une expérience, de leçons apprises depuis une pratique. Elles transforment ces savoirs en langage et en puissance d'agir. Elles permettent de charger le langage d'une performativité issue de connaissances écologiques, éthologiques, mystiques. Telle incantation permettra par exemple de faire venir tel poisson à tel endroit. Or à ce moment, l'incantation est aussi la traduction langagière d'un savoir, car le pêcheur connaît les habitudes de ce poisson, connaît aussi les courants du fleuve, la végétation et sait que ce poisson sort à tel moment de la journée, de l'année. L'incantation s'appuie sur ce savoir pour gagner en puissance.

Déclamer des incantations dans le pékâne c'est, en écho à l'énergie de l'eau, charger l'espace de la performance d'une puissance d'agir.

13. À Ngaolé, on m'a raconté ainsi que l'intronisation d'un nouveau Jaaltaabe (chef traditionnel des pêcheurs, le responsable local du fleuve) donnait lieu à une épreuve. Le futur Jaaltaabe doit ainsi réciter un certain nombre d'incantations face aux pêcheurs qu'il dirigera par la suite. 


\section{Tisser des cartes}

Les performances de pékâne sont composées comme des itinéraires qui font voyager l'auditoire d'un lieu à un autre, s'arrêtant parfois plus longuement à certaines étapes. Il s'agit d'un chant fortement toponymique et où les noms propres sont nombreux. L'extrait ci-dessous constitue un passage typique de pékâne :

doo njimnoomi e Mayra Sambayal Pinaa Demmba Abdul

ici j'ai chanté les louanges de Mayra Sambayal Pinâ Demba Abdoul ${ }^{14}$

nokku Sammba Mara Nyay

le lieu de Samba Mara Nyang ${ }^{15}$

mbayniimi e Beelinaabe

j'ai dit au revoir à Bêlinâbé ${ }^{16}$

doo ndewmi e Hayre Buubu Si

ici je suis passé sur la Colline-de-Boûbou-Si ${ }^{17}$

telliimi e Salnde Jumaa Pennda

je suis descendu au Carrefour-de-la-Mosquée-de-Penda

mbodo fooda nii gawde koole oole e nammaade

je me dirige vers les acacia nilotica, les mytragina inermis jaunes et les baubinia rufescens

tawnoomi ee Sara Aamadu Hammadi

j'ai rencontré Sara Amadou Hamadi ${ }^{18}$

oo ni Mariam Kebe

et aussi Mariam Kébé ${ }^{19}$

14. Mayra Sambayal Pinâ Demba Abdoul : hôtes de Souleymâne Sall, le poète, dans le village de Bêlinâbé où il se trouve.

15. Samba Mara Nyang : habitant du village de Bêlinâbé.

16. Bêlinâbé : village mauritanien situé au bord du fleuve Sénégal.

17. Colline-de-Boûbou-Si : grande dune de sable visible en sortant du village de Bêlinâbé.

18. Sara Amadou Hamadi : habitant du village de Gabâbé.

19. Mariam kébé : épouse de Sara Amadou Hamadi. 
njimnoomi e Ndayri Demmba Gay

j'ai chanté les louanges de Ndayri Demba Gaye ${ }^{20}$

doo miin mbayniimi e Gabaabe

ici j'ai dit au revoir à Gabâbé ${ }^{21}$

doo ndewmi e Ganki Farmal Haamiidu

ici je suis passé par le Micocoulier-de-Farmal-Hâmîdou ${ }^{22}$

L'extrait ci-dessus est représentatif de ce qui constitue une importante partie des performances de pékâne : la description de lieux et la mention des gens qui y habitent. Le poète passe d'un lieu à un autre en citant des personnes qu'il a rencontrées ou dont l'histoire fait partie du lieu. Ce genre d'enchaînement de lieux peut donner l'impression d'une forme d'hermétisme tant les noms propres y sont nombreux. Les lieux cités sont de plusieurs natures : villages (Bêlinâbé et Gabâbé), lieux-dits (Colline-de-Boûbou-Si, Carrefour-de-la-Mosquée-de-Penda), arbres remarquables (acacia nilotica, mytragina inermis, baubina rufescens, Micocoulier-de-Farmal-Hâmîdou), et les personnes sont nombreuses. Le poète passe en revue les lieux et les humains à grande vitesse. La structuration de cette description fait écho à la pratique des poètes et des pêcheurs : le mouvement. Durant leur apprentissage, les chanteurs réalisent des tournées (lappi en poulâr) au bord du fleuve, ils passent d'un village à un autre pour apprendre l'histoire des lieux et des hommes et éventuellement se former auprès d'autres chanteurs. Ils continuent plus tard à parcourir la région pour se produire. Les pêcheurs qui vivaient totalement de la pêche étaient eux aussi des nomades. Ils passaient l'hivernage dans leur village d'origine mais prenaient ensuite leurs pirogues pour suivre le poisson et ils vivaient d'un campement à un autre. La structure des chants reprend ce mode de vie et en fait un principe de composition.

Chaque vers ici représente une unité mélodique, un souffle à l'issue duquel le chanteur fait une courte pause. Dans cet extrait, plusieurs vers respectent le même schéma : verbe de mouvement à la première personne + nom de lieu et/ou nom de personne. Les verbes de mouvement sont majoritairement à l'accompli (telliimi, njiimoomi, mbayniimi, ndewmi, tawnoomi) mais un est à l'inaccompli (mbodo fooda), donnant l'impression que le mouvement passé se rejoue au présent grâce au chant. Ces mouvements sont ceux du poète : « je suis passé par », « je suis

20. Ndayri Demba Gaye : habitante du village Gabâbé.

21. Gabâbé : village sénégalais situé au bord du fleuve Sénégal, en face de Bêlinâbé.

22. Farmal Hâmidou : homme originaire de Gabâbé. 
LES MOTS EN CRUE : POÉSIE ET ÉCOLOGIE

descendu », « je me dirige vers ». Ces verbes font ressentir les reliefs traversés, les aspérités d'un territoire dont le point le plus bas est le fleuve auquel on accède en descendant parfois des berges escarpées, mais qui contient aussi des champs, des chemins qu'on emprunte pour aller à la rencontre d'habitants auxquels on dit adieu quelques mots plus tard. Le mouvement est le point de puissance du vers. Placés au début des vers, ces verbes de mouvement sont fortement accentués, l'arrivée dans un lieu qu'elle se fasse en marchant, en descendant ou en chantant correspond à une montée en intensité de la voix qui redescend ensuite pour décrire le lieu. La forte intensité de la voix souligne le mouvement qui crée la relation d'un lieu à un autre. La voix tisse les lieux, les lie les uns aux autres en rendant le mouvement sonore, accentué.

Ce tissage poétique a une dimension cartographique. Le chant reproduit l'itinéraire réel du chanteur, suit la réalité du territoire. L'ordre des toponymes dans le chant suit l'ordre géographique et dessine le chemin emprunté par le poète. Chaque itinéraire représente une séquence à l'issue de laquelle le poète peut faire une digression ou débuter un nouvel itinéraire. Cette façon particulière de tisser les lieux a un nom en poulâr : le jaraale. L'étymologie et la signification de ce terme varient selon les interlocuteurs. Selon les chasseurs ${ }^{23}$, le terme jaraale viendrait de la racine verbale yar- qui signifie boire (et le terme yaraango désignerait un lieu d'abreuvement). Selon certains pêcheurs, jaraale viendrait de la racine verbale jaar- qui signifie chanter (quelqu'un), louer, remercier (Seydou, 1998). Ces deux dimensions, point d'abreuvement et remerciement, sont bien présentes dans le pékâne. Le poète s'arrête dans les lieux de vie qui « pour la plupart » sont des points d'abreuvement (des animaux mais aussi des végétaux et des humains), et l'évocation de ces lieux est l'occasion de rendre hommage aux vies qui s'y déploient.

\section{Entremêler les mondes}

Faire lieu dans le pékâne, c'est parfois en détailler la complexité : s'y arrêter, en montrer les multiples dimensions, définir éventuellement des hauts lieux non par leur taille ou leur nombre d'habitants, mais par la richesse, l'intelligence et la sensibilité des dialogues qui s'y sont noués et s'y nouent toujours.

Plusieurs lieux donnent l'occasion de longs développements et permettent de saisir comment cette poétique des lieux est une poétique relationnelle qui permet

23. Information recueillie par mon collègue Aboubakry Sow, ethnomusicologue travaillant sur le keroone, la poésie des chasseurs de la même région. 
d'appréhender la façon dont les pêcheurs « façonnent des compréhensions de leur environnement, et les ressources conceptuelles au moyen desquelles ils constituent le monde qui les entoure et lui insufflent de la valeur et du sens » (Basso, 2016, p. 92). Chanter un lieu agit comme un déclencheur de mémoire, comme une porte ouverte sur des récits associés que la mention au lieu active pour l'auditeur averti.

Ainsi Ngaolé, petit village de pêcheurs au bord du fleuve Sénégal fait figure de haut-lieu sur lequel tous les poètes s'attardent longuement, comme Souleymâne Sall dans cet extrait d'une performance enregistrée en 2012 non loin de Ngaolé :

bamde e Nawle e yoral

j'ai commencé par Ngaolé juché sur la berge haute

doo ko leydi Muusa Bukari

ici, c'est le pays de Moûssa Boukari ${ }^{24}$

Yero Idi Isa Sammba Yetteenv

Yéro Idi Issa Samba Yétêne ${ }^{25}$

doon welii laamaade

là-bas, gouverner était agréable

diggu welii mundde

la farine était agréable en bouche

ndewmi e Kotaal e Falo Maram

je suis passé par Kotâl ${ }^{26}$ et le Jardin-au-bord-de-l'eau-Marame ${ }^{27}$

Njanku yoral ngal

$\mathrm{Njankou}^{28}$ de la berge haute

24. Moussa Boukary Sarr : fondateur du village.

25. Yéro Idy, Issa Samba Yétêne : parents de Moussa Boukary Sarr.

26. Kôtal : rivière.

27. Jardin situé près de la rivière Kôtal.

28. Champs faisant partie du village de Ngaolé. 
Njanku Muusa Bukari

Njankou de Moûssa Boukari

jakre e yoral ngal

la dépression de la berge haute

Joopbe e Mboojbe Maalbe

les Diop, Mbôdji, Maal

Seksekbe e Jaajaabe

Seck et Diâ

doo falo Mamadi Aadama Kummba Jaa Raasin

ici, le jardin au bord de l'eau de Mamadi Âdama Koumba Diâ Râcine ${ }^{29}$

ndewmi e Duunde Cammalle

je suis passé sur l'Îlot-des-Vieux-Arbres

doo maayo Jibi Joop

ici, le fleuve de Djibi Diop ${ }^{30}$

Njesi njiimi e caali

Ndiéssi ${ }^{31}$ surplombe des rivières

caali njiimi e caali

les rivières surplombent les rivières

daande gine e yombayru

au bord desquelles les oiseaux marabouts se rassemblent

$[\ldots]$

Toggere Bojel ndeeni

la Butte-Exondée-où-les-lièvres-se-sont-rassemblés

29. Mamadi Âdama Koumba Diâ.

30. Djiby Diop : pêcheur originaire d'un autre village qui venait pêcher à Ngaolé.

31. Ndiéssi : endroit où pêchait Djiby Diop. 


\section{koyli Sanaa falo taanum Ceerno}

sous le Koyli-de-Sanâ le Jardin-au-bord-de-l'eau-du-petit-fils-du-maître

\section{kolongal e galle Saarbe}

les champs de la maison des Sarr

La description de Ngaolé se déploie et détaille les relations qui s'y nouent. Le poète commence par nommer le fondateur du village Moussa Boukary Sarr, renommé dans toute la région pour avoir dompté un crocodile puissant nommé « Ngaari Nawle ${ }^{32}$ ». Moussa Boukary détenait un savoir reconnu qui lui permettait de commander aux hommes comme aux bêtes et de maîtriser les puissances invisibles du fleuve (génies). Pour honorer le fondateur du village, le chanteur cite ensuite ses ancêtres : « Yéro Idi Issa Samba Yétêne ». Moussa est un personnage hybride, un homme appartenant aux mondes humains, animaux et invisibles. Moussa Boukary Sarr a un pied dans chaque monde car il a développé une connaissance de chaque monde. Il connaît le comportement du crocodile, celui des hommes comme celui des génies. C'est cette connaissance qui lui permet d'être un diplomate au sens où l'emploie Baptiste Morizot :

«Diplomatie » provient du grec ancien (diploma), signifiant « plié en deux ». Le plié en deux, c'est celui qui se trouve à la frontière, contorsionné de telle manière à avoir une partie dans chaque camp, et qui ce faisant rend possible une communication, par le partage d'un code hybride : il constitue un interprète qui joue le rôle de membrane à l'interface entre deux entités hétérogènes. [...] Le diplomate est plié en deux, entre deux langages et deux ethos, entre deux systèmes d'intérêts : c'est ce qui le rend apte à être négociateur... (Morizot, 2016, p. 30)

Moussa est celui « qui grâce à ses connaissances » rend possible la communication entre les différents mondes qui constituent le fleuve. C'est l'espace de Ngaolé, l'environnement spécifique qui donne lieu à ce langage commun. Une anecdote l'illustre particulièrement. Ngaolé est un village au bord du fleuve et a longtemps vu circuler les bateaux de commerce, tout comme les pirogues de pêcheurs, les vedettes de l'administration (notamment coloniale) circuler. Or depuis Moussa Boukary Sarr, chaque bateau doit demander l'autorisation pour

32. Litt. « le mâle puissant de Ngaolé », le terme ngaari peut s'utiliser pour nommer tout animal ou homme dégageant une grande force : taureau, cheval, hippopotame. 
naviguer devant le village sous peine de risquer un naufrage. Les gens de Ngaolé « à la suite de leur fondateur » sont réputés pour leurs compétences magiques qui leur permettent de commander les forces du fleuve, de rendre le fleuve dangereux pour quiconque ignore ou défie leur autorité. Cette capacité diplomatique, cette aptitude à agir avec la diversité des mondes qui composent l'environnement est visible dans la description des espaces qui composent le village, lesquels sont toujours des espaces de rencontre inter-mondes. La toponymie, les descriptions de lieu rendent compte des forces en présence, la poésie nomme des relations.

Ainsi, la longue description de Ngaolé est l'occasion de passer en revue la diversité géomorphologique, végétale et animale des espaces qui composent ce village : types de berges, rivières et fleuve, champs et jardins (toggere, yoral, kolongal, falo, dunnde, caali), toponymes végétaux, animaux et humains (maayo Jibi Joop, toggere bojel ndeeni, Koyli Sanaa, Dunnde Camalle). Ces toponymes montrent comment les formes de vivant s'entrelacent : une berge est le refuge des lièvres ${ }^{33}$, les champs de décrue sont utilisés par la famille Sarr ${ }^{34}$, une partie du fleuve prend le nom d'un pêcheur, l'arbre sous lequel s'asseyait un villageois devient toponyme (Koyli Sanaa). Ces toponymes ouvrent l'imaginaire aux alliances interspécifiques à l'œuvre dans le village. Ainsi le koyli, mitragina inermis, qui donne son nom au lieu-dit « koyli Sanaa » est l'arbre redouté du crocodile :

Alla yoo nooro yidaa koyli

oh Dieu le crocodile n'aime pas le koyli

doo nganndee koyli yidaa nooro koyli pudii nder ladde

ici sachez que le koyli n'aime pas le crocodile le koyli est parti pousser en brousse

koyli mbiltii ngubiima ngabii mbasii mbadii mbaafur yaagoolooli

il a bourgeonné s'est bien développé, il a fait des jeunes pousses à la façon des yaagoo

33. Cette berge est une butte exondée sur laquelle les lièvres se réfugient lorsque l'eau s'engouffre dans les terres au moment de la crue. Cette butte devient donc La-Butte-où -les-lièvres-se-rassemblent/toggere Bojel Ndeeni.

34. Kolongal : champs collectifs à côté des villages inondés par la crue et donnant donc lieu après le retrait des eaux à des cultures de décrue. Le terme kolongal désigne à la fois un type géomorphologique et un usage social. Ce sont toujours des champs à côté des villages dont l'usage est très codifié. 


\section{nde koyli fey y a fertaama}

quand les graines de koyli ont germé, ses branches du koyli ont été élaguées

\section{doo koyli ngujaa nja66aa nja66itaa lelnaa e naange}

ici les branches de koyli ont été enduites, piétinées à plusieurs reprises et étendues au soleil

Si le crocodile redoute le koyli, c'est parce que ses branches sont utilisées pour réaliser les harpons qui serviront aux pêcheurs pour les chasser. Le koyli est l'allié de l'homme contre le crocodile. Ils ont une relation privilégiée, ils font alliance pour se mettre à l'abri du danger que représente le crocodile.

Nommer, c'est décrire, faire exister, mais c'est aussi créer des lieux communs aux humains, animaux, végétaux, autres qu'humains, une façon d'inscrire dans l'espace les relations nouées par le passé et celles qui continuent de se vivre.

\section{Conclusion}

L'écologie déployée dans le pékâne, fondée sur un mode d'écoute spécifique, est aujourd'hui mise en danger par les bouleversements forts qu'a connu le bassin versant du fleuve Sénégal depuis les années 60. Avec la proclamation de l'indépendance des États sénégalais, malien et mauritanien, le fleuve Sénégal est devenu une frontière. Il a été réduit institutionnellement à une ligne de séparation, divisant la communauté qui habitait les deux rives indistinctement en deux communautés étrangères, parlant la même langue, le poulâr, partageant la même culture mais n'étant plus associées aux mêmes pays. Or pour ces communautés, le fleuve n'est pas une ligne, une séparation ${ }^{35}$, mais un espace habité, complexe, dans lequel des vies se déploient. On ne fait pas que franchir une ligne en traversant le fleuve, on entre dans un espace habité en trois dimensions. La réduction cartographique et symbolique en une ligne nie ainsi les mondes qui co-habitent dans le fleuve (humains, animaux, végétaux, invisibles). Ce sont

35. « maayo wonaa keerol/Le fleuve n'est pas une frontière »: nom d'un collectif de rappeurs et d'activistes mauritaniens qui luttent contre la marginalisation des populations poulârophones par l'État mauritanien et pour une vision d'unité au sein des populations poulâr sénégalaises et mauritaniennes. 
ces mondes que les pêcheurs côtoient quotidiennement ${ }^{36}$. Un autre processus a contribué à modifier en profondeur cet écosystème. À la fin des années soixante, une sécheresse massive s'est abattue sur le Sahel, et l'eau s'est raréfiée à un point tel que certaines années, le fleuve n'est pas entré en crue et que le niveau d'eau extrêmement bas a permis par endroits de le traverser à gué. Cette sécheresse a duré une trentaine d'années, ravageant la vie animale et végétale. Des communautés d'arbres ont été décimées, des espèces animales ont disparu (Ndong, 1995 ; Lericollais, 1976), un exode rural massif a poussé de nombreux jeunes gens sur les routes de l'émigration (Bourlet \& Lorin, 2018). La réponse à cette sécheresse dévastatrice, mais aussi la volonté politique et économique d'accélérer un processus de transformation d'une agriculture essentiellement vivrière en une agriculture adaptée à une économie marchande, à laquelle de nombreux États africains ont dû adhérer à coup de plans d'ajustements structurels, fut une série de grands aménagements du territoire qui ont profondément bouleversé les écosystèmes et les façons dont les communautés rurales vivaient leur environnement. Souhaitant favoriser le développement d'une culture irriguée affranchie des irrégularités des crues, l'OMVS (l'Organisation pour la mise en valeur du fleuve Sénégal), sous la responsabilité des États frontaliers du fleuve Sénégal, a construit deux barrages : l'un en aval au Mali, le barrage hydroélectrique de Manantali dont l'objectif, en créant une retenue d'eau, est de pouvoir gérer artificiellement les crues; l'autre en amont au Sénégal à Diama, dont la fonction est de dessaler les eaux du fleuve et de permettre à de larges surfaces agricoles de profiter de l'eau du fleuve pour l'irrigation des cultures de canne à sucre, de riz, d'oignons, etc.

Ces deux barrages ont été mis en route en 1986 et 1988. Parallèlement, les périmètres irrigués se sont multipliés de part et d'autre du fleuve, de multiples digues et canaux ont été construits, enclosant des espaces auparavant parcourus par les troupeaux après les récoltes, détournant les chemins de l'eau de la crue, gorgeant les terres et les eaux de pesticides. Ces aménagements ont été réalisés à l'insu des pêcheurs qui, pourtant responsables jusque-là de l'eau du fleuve et

36. Cette vision d'un fleuve comme monde dans lequel on habite s'est confrontée à la vision institutionnelle de la ligne de façon très concrète. En 1989, résultant d'années de tension entre communautés mauritaniennes beydanes et noires, des affrontements violents ont eu lieu entre Sénégal et Mauritanie. Le fleuve est devenu une ligne de front. Pour les pêcheurs, vivre le fleuve comme un monde a eu parfois pour conséquence la mort. Les deux frères de mon collaborateur principal et ami, Ibrahima Maal, responsable des pêcheurs de Podor, sont morts tués par les balles que les militaires mauritaniens ont tiré depuis les rives alors qu'ils pêchaient sur le fleuve dans leurs pirogues. La frontière à ce moment-là a détruit la possibilité de vivre sur le fleuve en assassinant ses formes de vie. 
donc très bons connaisseurs de l'écosystème fluvial, n'ont pas été consultés. Cette marginalisation dans la prise de décision est allée de pair avec une paupérisation croissante, les poissons ayant décliné aussi bien en quantité qu'en diversité, ce dont les pêcheurs rendent responsable les barrages et les politiques d'aménagement du territoire.

Ce changement des conditions d'existence de tout un écosystème est omniprésent dans les discours des pêcheurs lors de leurs prises de parole publiques et lors des entretiens menés à l'occasion de mes enquêtes. Le barrage de Diama en aval du fleuve a supprimé les mouvements des marées hautes et basses (en poulâr fergo et nasgo) qui dictaient quotidiennement les mouvements, ce qui a déséquilibré le rythme de la vie du fleuve, comme en témoigne Ousmane Niang dans une prise de parole immédiatement après une performance de pékâne en 2019 : « Aujourd'hui, il n'y a plus de marée haute ni de marée basse, le fleuve ne fait que courir » ou Ibrahima Maal lors d'un entretien réalisé en 2018 : « Le fleuve ne bouge plus, il est mort ${ }^{37}$. » Ces prises de paroles fortes ne sont que très peu relayées politiquement et n'influent pas sur les prises de décisions. Les cultures irriguées s'intensifient, les terres sont vendues, des digues sont construites, l'eau est de plus en plus polluée. D'un point de vue écologique, le basculement paraît chaque jour un peu plus irréversible. Et pourtant, dans ce qui apparaît comme une catastrophe pour les pêcheurs, la poésie continue inlassablement de faire vivre un autre modèle, non en revendiquant ou critiquant le développement actuel de la région, mais en faisant vivre par les performances orales une autre façon de ressentir et d'agir avec le fleuve, une relation spécifique à l'eau.

Si le pékâne ne revendique rien, il affirme et fait exister un mode d'existence qui porte en son sein une force écologique subversive par rapport au développement actuel de la Vallée du Fleuve Sénégal. Le pékâne déploie une poétique écologique, une poétique relationnelle qui lie l'ensemble des vivants, les fait coexister et dialoguer. Il ne s'agit pas de dire que cette cohabitation est en soi pacifiste. Elle est bien souvent conflictuelle, mais ces conflits sont l'objet d'un dialogue, d'une négociation continue. Le langage poétique est l'écho de cette négociation et de ces dialogues interspécifiques. Ce qui se joue dans la vallée du fleuve Sénégal, outre la dégradation objective des conditions matérielles d'existence, c'est la possibilité d'un dialogue fondée sur une écoute, d'une vision écologique où le vivant dans sa diversité ne constitue pas des ressources mais bien des entités qui répondent, agissent et cohabitent. Contre le silence et l'immobilité, la poésie propose une

37. Phrase répétée à plusieurs reprises lors d'entretiens par le responsable des pêcheurs de Podor, Ibrahima Maal. 
LES MOTS EN CRUE : POÉSIE ET ÉCOLOGIE DANS LE BASSIN VERSANT DU FLEUVE SÉNÉGAL

énergie qui fait relation. Cette énergie porte en elle une capacité performative, une puissance d'agir.

\section{Bibliographie}

BAsso Keith, 2016, L'Eau se mêle à la boue dans un bassin à ciel ouvert : paysage et langage chez les Apaches occidentaux, Zones sensibles, Paris, 196 p.

Bourlet Mélanie \& Lorin Marie, 2018, “An Environmental History of Literary Resilience: 'Environmental Refugees' in the Senegal River Valley” in Journal of Postcolonial Writing, no 54 (6), pp. 821-834.

Haeckel Ernst Heinrich Philipp August, 1866, Generelle Morphologie Der Organismen. Allgemeine Grundzüge Der Organischen Formen-Wissenschaft, Mechanisch Begründet Durch Die von Charles Darwin Reformirte Descendenztheorie, von Ernst Haeckel, Drug und Verlag von Georg Remer, Berlin, $574 \mathrm{p}$.

FELD Steven, 1996, "Waterfalls of song: An Acoustemology of Place Resounding in Bosavi, New Guinea” in Feld Steven \& Basso Keith (eds.), Senses of Place, School of American Research Press, Sante Fe, New Mexico, pp. 91-135.

LeRicollais André, 1976, « La sécheresse et les populations de la vallée du Sénégal » in Lericollais André La Désertification au sud du Sahara, Nouvelles éditions africaines, Dakar, p. 111-116.

Morizot Baptiste, 2016, Les Diplomates : cohabiter avec les loups sur une autre carte du vivant, Éditions Wildproject (Coll. « Domaine Sauvage »), Paris, $320 \mathrm{p}$.

Ndiaye Oumar, 2005, « Les mutations du Pekaan, chants épiques des pêcheurs du Foûta-Tôro (Sénégal, Mauritanie) » in BAumgardT Ursula \& Derive Jean (dir.), Paroles nomades, Karthala, Paris, p. 221-229.

Ndong Jean-Batiste, 1995, « L'évolution de la pluviométrie au Sénégal et les incidences de la sécheresse récente sur l'environnement » in Revue de géographie de Lyon, no 70 (3), p. 193-198. 
Seydou Christiane, 1998, Dictionnaire pluridialectal des racines verbales du peul: peul-français-anglais/ A Dictionary of Verb Roots in Fulfulde Dialects, Karthale (coll. Dictionnaire et Langues), Paris, 952 p.

Sow Abdoul Aziz, 2009, La Poésie orale peule : Mauritanie-Sénégal ; essai de typologie et choix de textes, L'Harmattan (coll. Oralités), Paris, 418 p.

Sy Amadou Abel, 1978, Seul contre tous : deux récits épiques des pêcheurs du fouta toro, Les Nouvelles éditions Africaines, Dakar, Abidjan, 164 p.

Résumé : Lors d'une performance de pékâne, nom de la poésie des pêcheurs du fleuve Sénégal, l'écoulement de l'eau est un élément central. La crue, les maelströms, les courants sont à la fois des motifs rythmiques et poétiques, le signe d'une maîtrise de l'environnement fluvial mais aussi un mode de mise en relation entre humains et formes de vie habitant le fleuve. Cette poésie permet de comprendre comment pour ces populations, l'écoute est un mode de connaissance du fleuve.

Mots-clefs : poésie, écopoétique, fleuve Sénégal, pékâne.

\section{Swelling words: poetry and ecology in the River Senegal's watershed}

Abstract: During a Pékâne's performance, the name of River Senegal fishermen's poetry, the water flow is a central element. The presence of the flood, maelstroms, streams are rhythmical and poetical patterns, the manifestation of the fluvial environment's mastery, but also a way of establishing relationships between humans and life forms living in the river. This poetry shows how, for those people, listening is a way of knowing the river.

Keywords: poetry, ecopoetics, River Senegal, pékâne

\section{Notes sur l'auteur}

Marie Lorin est chercheuse contractuelle au Llacan (Langage, langues et cultures d'Afrique UMR 8135 CNRS-Inalco-EPHE), où elle poursuit ses recherche dans le cadre du projet EcoSen (pour une analyse écopoétique des littératures de la vallée du fleuve Sénégal). 